\title{
SINGLE-LOOK POLSAR STATISTICAL ANALYSIS USING FRACTIONAL MOMENTS OF POLARIMETRIC WHITENING FILTER
}

\author{
Salman Khan and Raffaella Guida
}

Surrey Space Centre, University of Surrey, Guildford, Surrey GU2 7XH, U.K.

\begin{abstract}
This paper proposes a new method of estimating the shape parameters of polarimetric singlelook complex compound distributions which model synthetic aperture radar data. The estimators derived from this method utilize fractional moments of polarimetric whitening filter, and can be derived for all commonly occurring distributions. They also exhibit low variance properties.
\end{abstract}

Index Terms - Fractional moments, polarimetric whitening filter, texture parameter estimation, polarimetric SAR

\section{INTRODUCTION}

Synthetic Aperture Radar (SAR) data are inherently probabilistic and as a result many statistical distributions have been proposed for their stochastic modeling. For polarimetric SAR (PolSAR) data, these distributions take different multivariate forms for singlelook complex (SC) and multilook complex (MC) data formats. In this regard, the scalar texture product model, which assumes that the observed signal is a product of two independent random variates, has played a vital role. One of them is called texture, which is a positive scalar random variable, while the other one is called speckle, which is a gaussian variate. Many texture distributions like gamma $(\gamma)$, inverse gamma $\left(\gamma^{-1}\right)$, generalized inverse gaussian (GIG), fisher $(\mathcal{F})$, beta $(\beta)$, and inverse beta $\left(\beta^{-1}\right)$ have been proposed in literature, resulting in $\mathcal{K}, \mathcal{G}^{0}, \mathcal{G}$, Kummer- $\mathcal{U}, \mathcal{W}$, and $\mathcal{M}$ compound distributions [1,2]. One key element for the utilisation of these pdfs is parameter estimation of the corresponding texture pdf. In this paper, we will concentrate on singlelook complex PolSAR data only. The estimation methods can be generally classified as 1) mono-pol, where the texture pdf parameters are estimated for each mono-pol channel separately, and the final polarimetric estimate is taken as the average of the mono-pol estimates e.g. method of fractional moments (MoFM), proposed for the $\mathcal{G}^{0}$ pdf by Frery et al. [3], and 2) polarimetric, where the full polarimetric information is utilised (including the covariance between channels) to estimate the parameters e.g. method of log cumulants (MoLC) for SC PolSAR data, proposed by Anfinsen in [4]. Under the scalar product model, it has been shown that MoLC for SC PolSAR data has better statistical properties than mono-pol estimators [4]. The MoLC requires expressions for the theoretical log cumulants (LC) of texture pdfs, which exist for all the above mentioned texture pdfs, except the GIG. The $\mathcal{G}$ pdf, resulting from GIG textures, is a very flexible distribution, and therefore its parameter estimation is of interest $[5,6]$. Recently, it has been shown that GIG LCs can still be numerically computed $[5,6]$. However, estimators with closed form expressions are more desirable. In this paper, we present an alternative polarimetric estimator based on fractional moments (FM) of polarimetric whitening filter (PWF).

\section{METHODOLOGY}

The PWF [7] was originally defined by using the sample covariance matrix (SCM) estimator. In [4], an alternative form of PWF was defined for non-gaussian clutter using the fixed point (FP) estimator of covariance matrix. We refer to the former as PWF and the latter as FP-PWF. Under product model decomposition, FP-PWF, $y$, can be decomposed into a texture term, $\tau$, and a speckle term, $Q$, as:

$$
\begin{aligned}
y & =\tau\left(\mathbf{x}^{H} \Sigma_{\mathrm{FP}}^{-1} \mathbf{x}\right) \\
& =\tau Q,
\end{aligned}
$$

whose asymptotic statistics were derived in [4], where $(\cdot)^{H}$ is the Hermitian transpose, and $\mathbf{x}$ is the speckle vector. The FPPWF speckle term follows a Fisher variate, with known log cumulant expressions. Hence, MoLC for SC PolSAR data was applied for the $\mathcal{K}$ distribution [4].

Our first estimator, proposed here, is based on FMs of FPPWF and is called method of multivariate FMs (MoMFMFP). We strictly assume that the scattering vector is zero mean so that the raw moments of FP-PWF are also its central moments. Knowing the distribution of the speckle term of FPPWF from [4], we can easily find its FMs. Hence the FMs of FP-PWF are given by:

$$
\begin{array}{r}
\mathbf{E}\left\{y^{\nu}\right\}=\frac{\mathbf{E}\left\{\tau^{\nu}\right\}}{\mathbf{E}\{\tau\}^{\nu}}\left(\operatorname{det}\left(\Sigma_{\mathrm{FP}}\right)^{1 / d} \frac{N d}{(d+1)} \frac{(N-d-1)}{(N-d+1 / d)}\right)^{\nu} \\
\times \frac{\Gamma(d+\nu)}{\Gamma(d)} \frac{\Gamma\left(\frac{d(N-d+1 / d)}{d+1}-\nu\right)}{\Gamma\left(\frac{d(N-d+1 / d)}{d+1}\right)},
\end{array}
$$


where $\operatorname{det}\left(\Sigma_{\mathrm{FP}}\right)^{1 / d}$ appears because $\Sigma_{\mathrm{FP}}$ is normalized such that its determinant is equal to unity. The $d$ represents dimension, and $N$ is the sample size.

For the $\mathcal{K}$ distribution, we can easily compute the $\frac{\mathbf{E}\left\{\tau^{\nu}\right\}}{\mathbf{E}\{\tau\}^{\nu}}$ ratio of $\gamma$ texture pdf given by:

$$
\frac{\mathbf{E}\left\{\tau^{\nu}\right\}}{\mathbf{E}\{\tau\}^{\nu}}=\frac{\Gamma(\alpha+\nu)}{\alpha^{\nu} \Gamma(\alpha)}
$$

The $\mathcal{K}$ distribution has only one shape parameter, $\alpha>0$, inherited from its $\gamma$ texture pdf. Thus, we need to solve only one equation derived by putting (3) in (2) with $\nu=1 / 8$.

We can also derive the distribution of speckle term for PWF. The derivation is very similar to that of FP-PWF in [4]. Hence the FMs of PWF are given by:

$$
\begin{aligned}
\mathbf{E}\left\{y^{\nu}\right\}= & \frac{\mathbf{E}\left\{\tau^{\nu}\right\}}{\mathbf{E}\{\tau\}^{\nu}}\left(\operatorname{det}(\Sigma)^{1 / d} \frac{N(N-d)}{N-d+1}\right)^{\nu} \\
& \times \frac{\Gamma(d+\nu)}{\Gamma(d)} \frac{\Gamma(N-d+1-\nu)}{\Gamma(N-d+1)},
\end{aligned}
$$

Hence, we can define our second estimator, which is based on FMs of PWF (not FP-PWF), and is called MoMFM. Since we can derive the speckle distribution of PWF as:

$$
Q_{S C M} \stackrel{N \rightarrow \infty}{\sim} \frac{N d}{N-d+1} \mathcal{F}_{1, d, N-d+1},
$$

we can also list an MoLC based estimator using PWF (not FP-PWF), denoted as MoLC estimator with the speckle log cumulants:

$$
\begin{aligned}
\kappa_{1}\left\{Q_{S C M}\right\} & =\psi^{(0)}(d)-\psi^{(0)}(N-d+1) \\
& +\ln \left(\frac{N(N-d)}{N-d+1}\right) \\
\kappa_{\nu>1}\left\{Q_{S C M}\right\} & =\psi^{(\nu-1)}(d) \\
& +(-1)^{\nu} \psi^{(\nu-1)}(N-d+1) .
\end{aligned}
$$

For $\mathcal{K}$ distribution, we also have two other estimators from literature: 1) one based on multivariate kurtosis [8], abbreviated as method of multivariate kurtosis (MoMK), and 2) mono-pol MoFM [4].

We can compare all the above mentioned estimators for the $\mathcal{K}$ distribution using simulated singlelook PolSAR data. Figure 1 shows the pdfs of estimator bias after 5000 Monte Carlo simulations with sample size 1024, simulated with $\alpha=$ 5. Table 1 shows the $p$ values of fitting normal (norm) and generalized extreme value (gev) distributions to bias pdfs using $\chi^{2}$ goodness-of-fit (GoF). This is done to accurately calculate the mean and variance of the bias pdf (shown in table 1) using the mean and variance of norm or gev, whichever has a higher $\mathrm{p}$ value. Table 1 shows that $\alpha_{\text {MoMFM }}$ bias exhibits the second lowest mean greater than only $\alpha_{\mathrm{MoMFM}}(\mathrm{FP})$, and the second lowest variance after $\alpha_{\text {MoMFM }}(\mathrm{FP})$. Note that FM of $\frac{1}{8}$ was used.

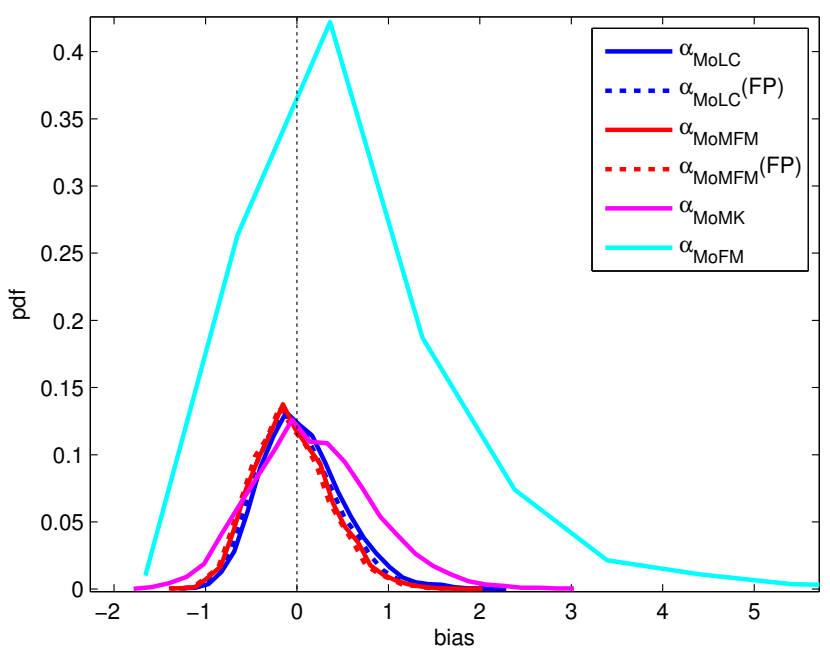

Fig. 1. PDF of estimator bias for polarimetric singlelook $\mathcal{K}$ distribution at sample size $=1024$, and true value $\alpha=5$.

Table 1. Mean and variance of empirical pdfs of fig. 1.

\begin{tabular}{|l|l|c|c|c|}
\hline \multicolumn{1}{|c|}{ Estimator } & $p_{\text {norm }}$ & $p_{\text {gev }}$ & $\mu$ & $\sigma^{2}$ \\
\hline \hline$\alpha_{\text {MoLC }}$ & 0 & 0.08 & 0.066 & 0.210 \\
\hline$\alpha_{\text {MoLC }}(\mathrm{FP})$ & 0 & 0.24 & 0.004 & 0.200 \\
\hline$\alpha_{\text {MoMFM }}$ & 0 & 0.86 & 0.042 & 0.185 \\
\hline$\alpha_{\text {MoMFM }}(\mathrm{FP})$ & 0 & 0.87 & 0.073 & 0.180 \\
\hline$\alpha_{\text {MoMK }}$ & 0 & 0.05 & 0.176 & 0.440 \\
\hline$\alpha_{\text {MoFM }}$ & 0 & 0 & 0.604 & 1.915 \\
\hline
\end{tabular}

The usefulness of the new estimator can also be observed by its application to the $\mathcal{G}$ distribution, where applying MoLC is a challenge since no closed form expressions exist for the LCs of GIG pdf. It should be noted that the MoLC based estimators for $\mathcal{G}$ distribution can still be applied as shown by the authors in [6], using numerical techniques. The $\mathcal{G}$ distribution has two texture shape parameters $\alpha \in \mathbb{R}$ and $\omega>0$. Its $\frac{\mathbf{E}\left\{\tau^{\nu}\right\}}{\mathbf{E}\{\tau\}^{\nu}}$ ratio is given by:

$$
\frac{\mathbf{E}\left\{\tau^{\nu}\right\}}{\mathbf{E}\{\tau\}^{\nu}}=\frac{K_{\alpha+\nu}(\omega) K_{\alpha}^{\nu-1}(\omega)}{K_{\alpha+1}^{\nu}(\omega)},
$$

which can be put in (2). Consequently, two equations are solved simultaneously for MoMFMs based estimators using FMs of $\frac{1}{8}$ and $\frac{1}{4}$. Figure 2 shows the estimator bias pdfs for $\mathcal{G}$ distribution after 5000 Monte Carlo simulations with sample size 1024 at $\alpha=5, \omega=5$. We have not shown the monopol MoFM estimator bias pdf as it has a very high variance. The bias pdfs are multi-modal, hence we have used gaussian mixture model (gmm) to compute their means and variances. Figure 3 shows an example of this fitting for $\alpha_{\text {MoLC }}$ where both the norm and gev poorly fit the bias pdf, while gmm shows an excellent fit with a $\chi^{2}$ GoF p value of 0.98 . Table 2 shows the means and variances of the bias pdfs. It is clear that 

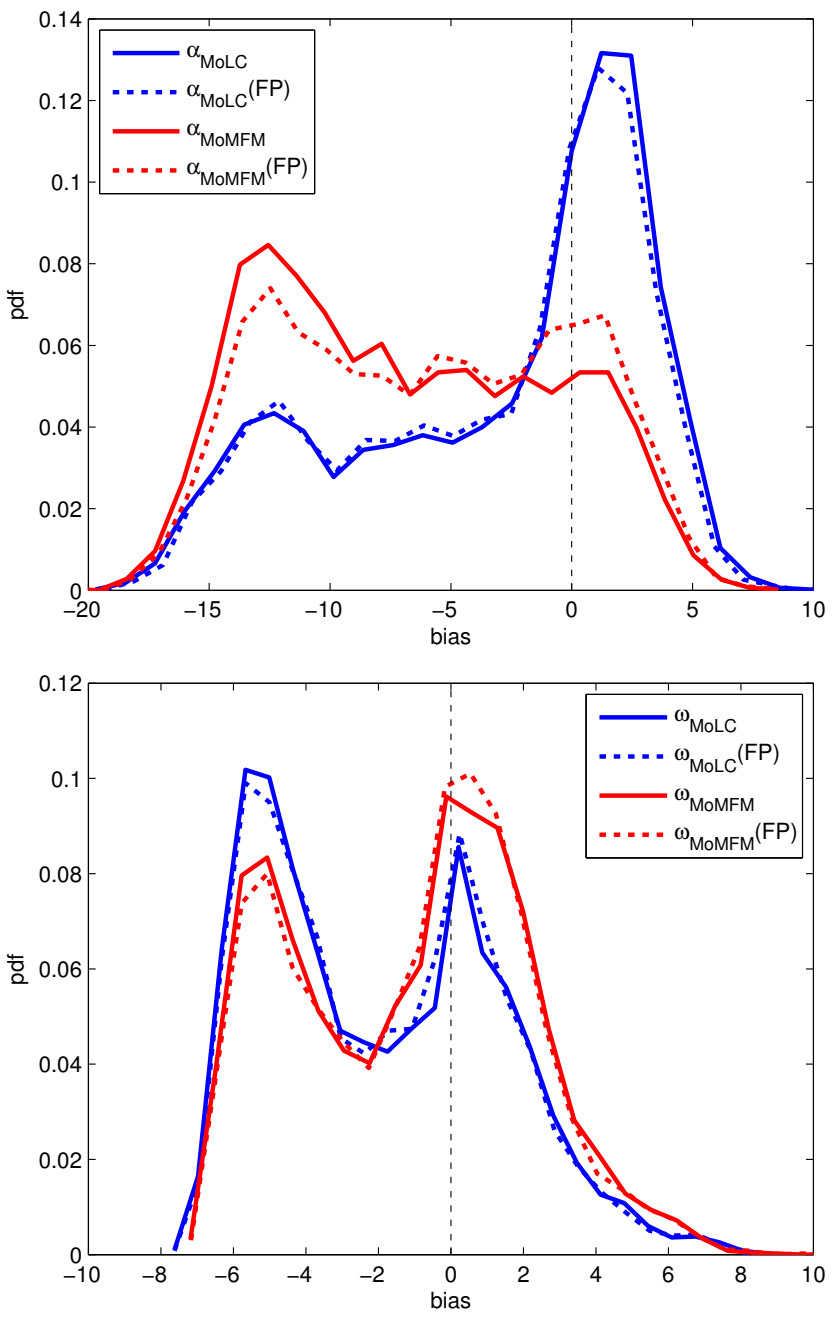

Fig. 2. PDF of estimator bias for (Left) $\alpha$ and (Right) $\omega$ parameters of polarimetric singlelook $\mathcal{G}$ distribution at sample size $=1024$, and true value $\alpha=5, \omega=5$.

gmm fits the bias pdfs very well (shown by higher $\mathrm{p}$ values). The mono-pol MoFM estimators perform the worst shown by extremely high variance. For $\alpha$ parameter, although MoMFM based estimators show higher bias than MoLC based estimators, they have lower variance. However, for $\omega$ parameter MoMFM (FP) exhibits the lowest bias and variance. The proposed estimators can therefore be regarded as a valuable alternative to MoLC based estimators.

\section{CONCLUSIONS}

In this paper, we have proposed an alternative method of estimating the texture shape parameters of SC PolSAR distributions with the example of $\mathcal{K}$ and $\mathcal{G}$ distributions. The proposed estimators are based on FMs of PWF and FP-PWF. The mathematical forms of the estimators are also included. Ex-

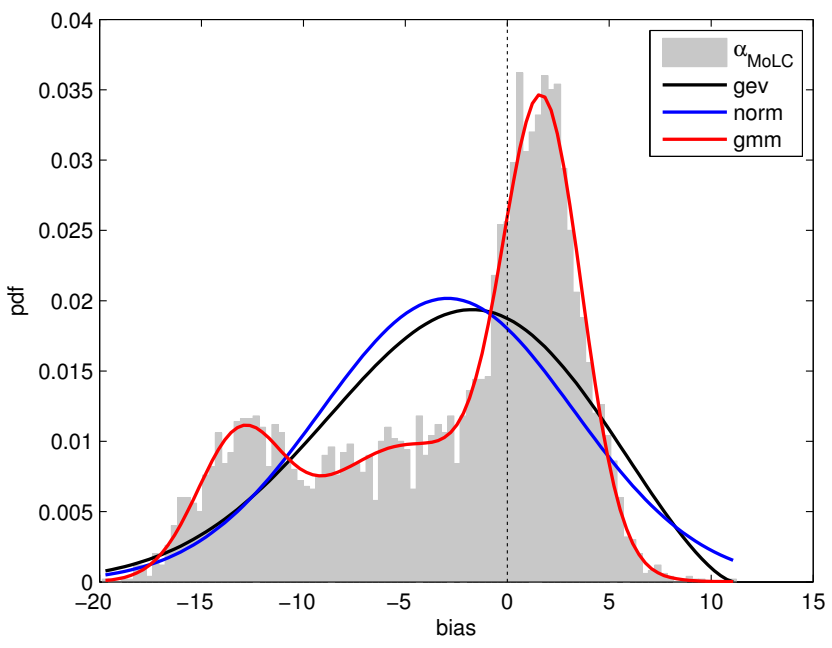

Fig. 3. Fitting of gmm to empirical pdf of $\alpha_{\mathrm{MoLC}}$ bias, with a $\mathrm{p}$ value of 0.98 using $\chi^{2} \mathrm{GoF}$.

Table 2. Mean and variance of empirical pdfs of fig. 2.

\begin{tabular}{|l|l|l|l|c|c|}
\hline \multicolumn{1}{|c|}{ Estimator } & $p_{\text {norm }}$ & $p_{\text {gev }}$ & $p_{\text {gmm }}$ & $\mu$ & $\sigma^{2}$ \\
\hline \hline$\alpha_{\text {MoLC }}$ & 0 & 0 & 0.98 & -2.930 & 38.136 \\
\hline$\alpha_{\text {MoLC }}(\mathrm{FP})$ & 0 & 0 & 0.82 & -3.122 & 36.676 \\
\hline$\alpha_{\text {MoMFM }}$ & 0 & 0 & 0.98 & -6.901 & 34.621 \\
\hline$\alpha_{\text {MoMFM }}(\mathrm{FP})$ & 0 & 0 & 0.79 & -5.989 & 35.032 \\
\hline$\alpha_{\text {MoFM }}$ & 0 & 0 & 0.12 & -0.548 & $9.067 \mathrm{e}+3$ \\
\hline$\omega_{\text {MoLC }}$ & 0 & 0 & 0.83 & -1.987 & 10.532 \\
\hline$\omega_{\text {MoLC }}(\mathrm{FP})$ & 0 & 0 & 0.37 & -1.943 & 10.212 \\
\hline$\omega_{\text {MoMFM }}$ & 0 & 0 & 0.32 & -1.181 & 10.471 \\
\hline$\omega_{\text {MoMFM }}(\mathrm{FP})$ & 0 & 0 & 0.37 & -1.110 & 10.164 \\
\hline$\omega_{\text {MoFM }}$ & 0 & 0 & 0 & 1.141 & $5.891 \mathrm{e}+3$ \\
\hline
\end{tabular}

perimental results on simulated PolSAR data show that the proposed estimators exhibit the lowest variance and also their bias is second to only MoLC based estimators. Thus for PolSAR distributions, which do not have closed form LC expressions, MoMFM based estimators can serve as a valuable alternative.

\section{REFERENCES}

[1] L. Bombrun, S. Anfinsen, and O. Harant, "A complete coverage of log-cumulant space in terms of distributions forpolarimetric SAR data," in Proc. PolInSAR, Frascati, Italy, 2011.

[2] S. Khan and R. Guida, "On single-look multivariate $\mathcal{G}$ distribution for PolSAR data," IEEE J. Sel. Topics Appl. Earth Observations Remote Sens., vol. 5, no. 4, pp. 1149 -1163, Aug. 2012.

[3] A.C. Frery, H.-J. Muller, C.C.F. Yanasse, and S.J.S. 
Sant' Anna, "A model for extremely heterogeneous clutter," IEEE Trans. Geosci. Remote Sens., vol. 35, no. 3, pp. 648-659, May 1997.

[4] S. Anfinsen, "On the supremacy of logging," in Proc. PolInSAR, Frascati, Italy, 2011.

[5] S. Khan and R. Guida, "The new dual-texture $\mathcal{G}$ distribution for single-look PolSAR data," in Proc. IGARSS, Munich, Germany, Jul. 2012, in press.

[6] S. Khan and R. Guida, "Application of Mellin kind statistics to polarimetric G distribution for SAR data," IEEE Trans. Geosci. Remote Sens., 2012, submitted 28-Nov2012.

[7] L.M. Novak, M.C. Burl, and W.W. Irving, "Optimal polarimetric processing for enhanced target detection," IEEE Trans. Aerosp. Electron. Syst., vol. 29, no. 1, pp. 234-244, Jan 1993.

[8] A.P. Doulgeris and T. Eltoft, "Scale mixture of Gaussians modelling of polarimetric SAR data," EURASIP J. Adv. Signal Process, vol. 2010, no. 874592, pp. 1-12, Jan. 2010. 